\title{
Performance of Sprayed Fiber Reinforced Polymer Strengthened Timber Beams
}

\author{
S. Talukdar and N. Banthia \\ Department of Civil Engineering, University of British Columbia, 6250 Applied Science Lane, Vancouver, BC, Canada V6T $1 Z 4$ \\ Correspondence should be addressed to S. Talukdar, sudip@civil.ubc.ca
}

Received 26 July 2010; Revised 8 October 2010; Accepted 12 October 2010

Academic Editor: Krystyn J. Van Vliet

Copyright (๑) 2010 S. Talukdar and N. Banthia. This is an open access article distributed under the Creative Commons Attribution License, which permits unrestricted use, distribution, and reproduction in any medium, provided the original work is properly cited.

A study was carried out to investigate the use of Sprayed Fiber Reinforced Polymer (SFRP) for retrofit of timber beams. A total of 10-full scale specimens were tested. Two different timber preservatives and two different bonding agents were investigated. Strengthening was characterized using load deflection diagrams. Results indicate that it is possible to enhance load-carrying capacity and energy absorption characteristics using the technique of SFRP. Of the two types of preservatives investigated, the technique appears to be more effective for the case of creosote-treated specimens, where up to a $51 \%$ improvement in loadcarrying capacity and a $460 \%$ increase in the energy absorption capacity were noted. Effectiveness of the bonding agent used was dependent on the type of preservative the specimen had been treated with.

\section{Introduction}

Changing societal needs, upgrading of design standards, increased safety requirements, and deterioration renders concrete, masonry, and timber structures such as bridges and buildings structurally deficient or functionally obsolete and need to be replaced or upgraded through strengthening [1]. Structures which have been excessively deteriorated may have undergone severe strength loss and pose a serious threat to public safety. Therefore, it is imperative that structures be routinely inspected and any loss of capacity due to damage/deterioration be promptly addressed, so as to avoid having to replace the structure or the event of a catastrophic failure.

Disadvantages associated with traditional rehabilitation/retrofit techniques have led researches to develop new techniques, such as use of Fiber Reinforced Polymers (FRPs). FRP offers an excellent combination of mechanical and physical properties, including low weight, immunity to corrosion, ability to form long lengths, and excellent specific strength/stiffness.

A new technique developed at the University of British Columbia (UBC) allows for application of FRP via spray. Prior to application, the element surface is coated with a bonding agent/adhesive. Polymer is then sprayed out of a nozzle towards the surface. Attached to the nozzle is a fibre chopping device, which cuts the fibre into desired lengths and injects it into the spray stream along with the polymer. The FRP then impacts the surface and coats it. A major advantage of this system is that the fibres are oriented in a $2 \mathrm{D}$ random manner in the plane of placement. The advent of SFRP as an application method allows for FRP to be applied in a less labor-intensive and more cost-effective manner.

Furthermore, when comparing FRP to SFRP, it is virtually impossible to fully engage unidirectional FRP in tension and achieve its tensile strength, as typically the FRP will debond from the substrate surface before full FRP strength is achieved. The randomly oriented SFRP achieves a better bond with the substrate surface due to the following reasons [2]:

(1) lack of fiber to fiber continuity ensures that the composite does not debond as a continuum;

(2) the process allows for a better bond due to high pressure pneumatic compaction, the energy absorption capacity is higher, and the FRP itself is more damage tolerant; 
(3) there is better dimensional compatibility with the substrate as the elastic moduli of the substrate and the repair material are similar causing lower interfacial stresses and a more favorable morphology.

Structural timber members can be easily damaged by poor maintenance practices and surface degradation due to overloading, pests, or weather conditions over years of use. Worldwide, there are thousands of timber bridges in service, which will inevitably require replacement/retrofit. According to the 2005 "Report Card for Americas Infrastructure" [3], there are 160570 bridges in the United States which are classified as structurally deficient. Approximately $7 \%$ of the national bridge inventory consists of timber bridges. If the same ratio of structurally deficient bridges applies to the timber bridge stock, this would mean well over 10000 timber bridges in the USA are structurally deficient. Instead of replacing the existing structures, applying SFRP reinforcement to the existing structures could be a more costeffective solution.

The main objective of this study was to investigate whether or not application of SFRP to timber members is a feasible rehabilitation technique.

\section{Previous Work}

While the application of SFRP to timber is an entirely new technique, FRP composites have been studied and used in the past to successfully reinforce timber structures. Research on timber beams reinforced with FRP materials has increased beginning in the 1990s. Plevris and Triantafillou [4] conducted an experimental and numerical investigation of beams reinforced with Carbon Fiber Reinforced Polymer (CFRP) sheets and concluded it was possible to increase the load-carrying capacity of a specimen by increasing the area fraction of the fiber composite to an upper limit. Gentile [5] looked at the flexural strengthening of timber bridge beams using Glass Fiber Reinforced Polymer (GFRP) rods. He found it was possible to increase the flexural strength and ductility using such a method. Dempsey and Scott [6] evaluated the effect of mechanically fastening FRP strips to Southern pine wood members and concluded it was possible to increase ultimate moment, stiffness, and ductility. The magnitude of each increase was partially dependant on fastener spacing. Johns and Lacroix [7] looked at the application of GFRP and CFRP to commercial sawn lumber specimens. They concluded that the application of the strengthening material increases the effective strength of the wood and has a greater effect on increasing strength of smaller, lower grade sections. Fiorelli and Dias [8] conducted similar tests on pinewood beams and compared their results to a theoretical model. They noted that use of reinforcement leads to an increase in ductility, particularly in the failure phase of a test.

Additionally, there have been numerous tests carried out on laminated timber beams. Gilfillan et al. [9] investigated reinforced Sitka Spruce laminated beams. Dagher et al. [10] investigated the benefits of reinforcing glulam beams made with Eastern Hemlock. Johnsson et al. [11] looked at the strengthening of glulam members by CFRP bars. Issa and Kmeid [12] examined glulam beams reinforced with steel plates as well as CFRP. Dorey and Cheng [13] and Hernandez et al. [14] conducted experiments on reinforced glulam, with the common finding between all these studies being that FRP materials can increase strength and ductility of glulam beams and better utilize the compression strength of wood, by forcing failure in the compression zone at the top of the beam first, propagation of the failure downwards, and finally followed by eventual rupture in the tensile zone and failure of the beam. Consequently, the mode of failure in reinforced specimens was a ductile one while failure was sudden and brittle in unreinforced specimens.

Finally, it should be noted that in addition to laboratory studies of timber-FRP composites, there have been successful field applications. The 39-year-old Tourond Creek Bridge in Manitoba, Canada was the first timber bridge to undergo strengthening via reinforcement with GFRP bars. After rehabilitation, the 3-span, 23.3-meter structure is estimated to be at least $30 \%$ stronger. The province of Manitoba owns approximately 575 similar bridges, the majority of which are deficient in their load-carrying capacity by today's standards. It is estimated that bridges similar to the Tourond Creek Bridge can be strengthened for about $15 \%$ of the cost estimated to completely replace it [15].

\section{Experimental Program}

3.1. Specimen Preparation. The beam species selected was \#2 Douglas Fir, procured from a local building materials supplier. Each beam was first sanded with a medium-grit (\#60) belt sander, with the dual purpose of smoothening the surface to enhance wettability and to remove any contaminants. A total of ten specimens were tested of two different sizes. Specimens A-E were treated with Boracol preservative. These beams had dimensions of $150 \mathrm{~mm} \times$ $350 \mathrm{~mm} \times 2440 \mathrm{~mm}$. Beams F-J were treated with Creosote preservative. They had slightly different dimensions of $150 \mathrm{~mm} \times 300 \mathrm{~mm} \times 2440 \mathrm{~mm}$.

Boracol is a water-based wood preservative which is fungicidal and insecticidal with an added preventative mouldicidal effect. The second type of preservative is the commonly used oil-borne preservative known as Creosote. In addition to killing wood-destroying organisms, Creosote increases the dimensional stability of wood. Oil residue from creosote can also prevent the formation of chemical bonding with applied adhesives [16]. After the surfaces had been sanded and brushed clean, five of the beams were coated with Boracol at the rate of $4.5 \mathrm{~m}^{2} / \mathrm{l}$. The beams were then allowed to sit for 10 days to allow for subsurface penetration of the liquid. Five more beams were pressure treated with creosote at an off-site plant and then transported to the laboratory for testing.

For this study, bond enhancement was achieved primarily through the use of chemical bonding agents. Two different types of adhesives/bonding agents were used: AtPrime 2 and HMR. AtPrime 2 is a 2-part primer resin developed by Reichold Inc. which serves to enhance secondary bonding performance [17]. Hydroxymethylated Resorcinol (HMR) is 


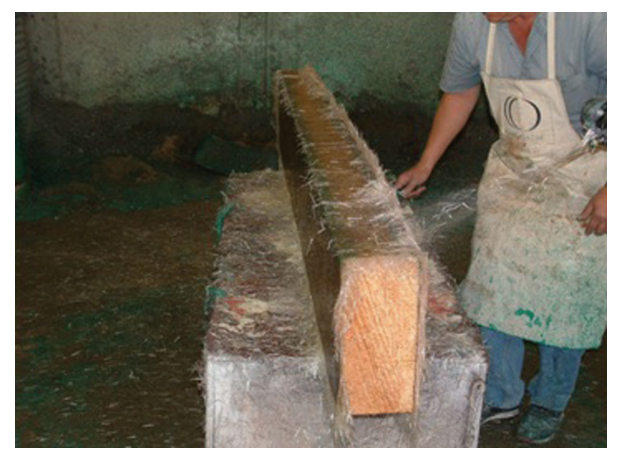

FIGURE 1: SFRP application.

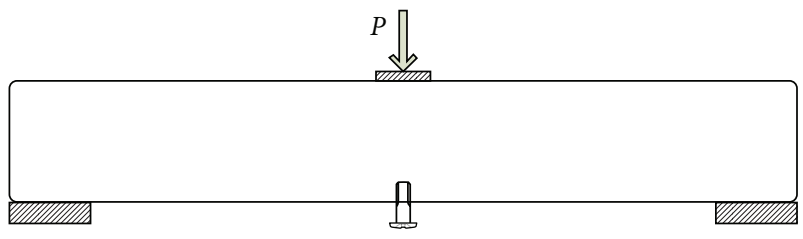

FIgURE 2: Beam dimensions and instrumentation diagram.

a primer/bonding agent developed in the 1990s at the USDA Forest Service Products Laboratory. It consists of mono-, di-, and trihydroxymethylated resorcinol and oligomers of such molecules. HMR was synthesized in the lab before application using the procedure described by Vick [18] and applied at the rate of $6.9 \mathrm{~m}^{2} / 1$. Studies confirming the various beneficial properties of HMR as a bonding agent were conducted by Christiansen and Vick [19], Lyons and Ahmed [20], and Richter and Steiger [21]. Prior to SFRP application, several of the test specimens were treated with either/both AtPrime 2 and HMR.

For SFRP, the type of resin used throughout was the 7136674 general-purpose unsaturated polyester resin manufactured by Hexion Specialty Chemicals. The type of fiber used throughout the experiment was ER2400JP6 Gun Roving, manufactured by Gibson Fiberglass. The FRP spray system is fairly straightforward, and the exact spray procedure is described by Banthia and Boyd [22] and shown in Figure 1. In practice, only 3 faces of any girder would be accessible, and hence, in testing, only 3 faces were sprayed. The thickness of spray applied on all retrofitted specimens was $6 \mathrm{~mm}$. Table 1 describes the test program.

The elastic modulus of the SFRP can be found in Solemani [23]. Solemani had conducted tests on SFRP coupons identical in composition to the FRP spray that was used throughout this project. After analyzing the stress-strain response of SFRP coupons subjected to direct tensile tests, Solemani concluded that the elastic modulus of the cured SFRP was $14 \mathrm{GPa}$.

3.2. Testing. The authors acknowledge the relatively limited number of specimens tested. However, since these tests were full scale and because the primary purpose was to compare the performance of retrofitted specimens with unretrofitted controls, data are both useful and applicable. Furthermore,

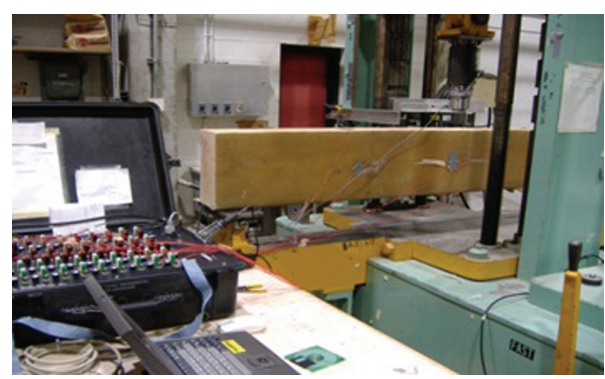

Figure 3: Test setup.

TABLE 1: Specimen testing program.

\begin{tabular}{lccc}
\hline & SFRP & Bonding agent & Preservative \\
\hline Specimen A & No & No & Boracol \\
Specimen B & Yes & No & Boracol \\
Specimen C & Yes & AtPrime only & Boracol \\
Specimen D & Yes & AtPrime + HMR & Boracol \\
Specimen E & Yes & HMR only & Boracol \\
Specimen F & No & No & Creosote \\
Specimen G & Yes & No & Creosote \\
Specimen H & Yes & AtPrime only & Creosote \\
Specimen I & Yes & AtPrime + HMR & Creosote \\
Specimen J & Yes & HMR only & Creosote \\
\hline
\end{tabular}

for the un-retrofitted controls, Canadian Wood Council Lumber Properties Research Project [24] predicts a significantly lower strength for the Boracol-treated specimen of this size and grade than measured in the program. For the Creosote-treated specimens, on the other hand, the predicted strength values are nearly the same as the measured ones [25]. Quasi-Static Testing was carried out on an $890 \mathrm{kN}$ Tinius Olsen's Hydraulic Loading Machine in a 3-point bending configuration.

Each beam was instrumented as shown in Figures 2 and 3. Deflection of the beam was measured using a Displacement Pod attached to the underside of the beam. The Displacement Pod model was a DT-40A unit manufactured by Dalimar Industries, having a data acquisition frequency of $1 \mathrm{~Hz}$.

\section{Results and Discussion}

4.1. Boracol-Treated Beams. As seen in Figure 4 and Table 2, application of SFRP can result in increases in beam ductility, toughness, and ultimate load-carrying capacity. It was observed that in the cases where SFRP ruptured, it was immediately followed by timber rupture. Based on Table 2, it is possible to infer that HMR has the effect of significantly increasing the ductility and toughness of SFRP strengthened beams. Beams D and E, both of which were treated with HMR showed gains in energy absorption of $100 \%$ and $83 \%$, respectively. In addition to simply trying to increase the flexural or shear strength of a beam, it may be equally important to increase ductility for improved seismic performance. Given that the HMR-treated specimens 
TABLE 2: Summary of results: boracol treated.

\begin{tabular}{|c|c|c|c|c|c|c|}
\hline & $\begin{array}{c}\text { Ultimate load } \\
(\mathrm{kN})\end{array}$ & $\begin{array}{l}\text { Ultimate displacement } \\
(\mathrm{mm})\end{array}$ & $\begin{array}{c}\text { Energy absorbed } \\
(\mathrm{kJ})\end{array}$ & $\begin{array}{c}\text { Load \% } \\
\text { increase over A }\end{array}$ & $\begin{array}{c}\text { Energy absorbed \% } \\
\text { increase over A }\end{array}$ & FRP rupture \\
\hline Beam A & 184.7 & 20.1 & 2.4 & - & - & - \\
\hline Beam B & 210.1 & 22 & 2.1 & $13.8 \%$ & $-12.5 \%$ & No \\
\hline Beam C* & 181.4 & - & - & $-1.8 \%$ & - & No \\
\hline Beam D & 215.2 & 31.6 & 4.8 & $16.5 \%$ & $100.0 \%$ & Yes \\
\hline Beam E & 215.4 & 30.1 & 4.4 & $16.6 \%$ & $83.3 \%$ & Yes \\
\hline
\end{tabular}

${ }^{*}$ Due to a data acquisition failure, displacements, and strain gauge readings from Beam $\mathrm{C}$ were not properly recorded.

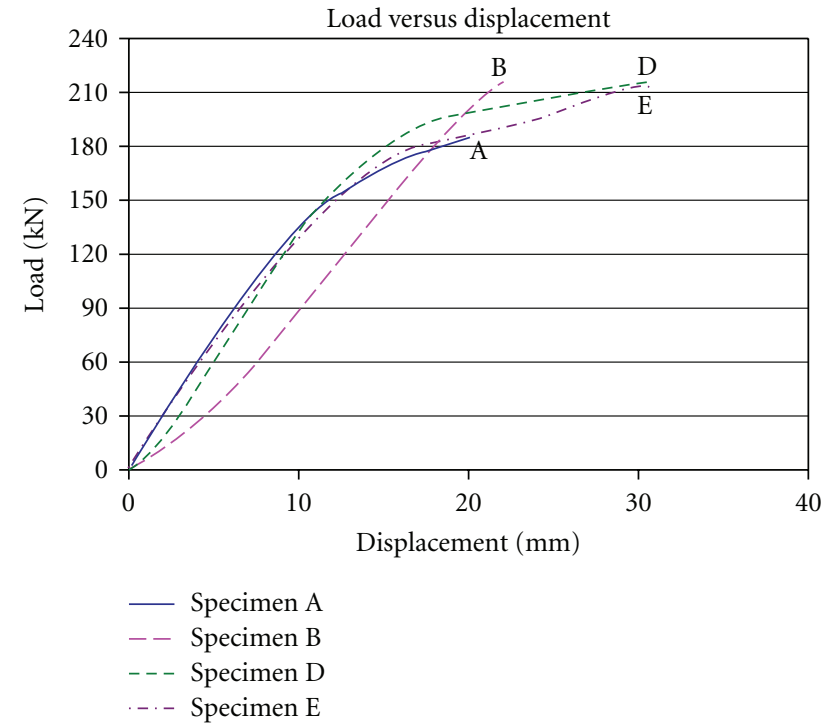

FIGURE 4: Comparison of results: boracol treated.

ultimately failed through SFRP rupture, use of HMR seems to promote composite action in these beams.

Use of SFRP on its own (Beam B) without the aid of any sort of bonding agent/primer provided an increase in flexural capacity, but the ductility/toughness did not increase at all. In this case, the SFRP did not rupture, indicating that a full composite action was not achieved. Therefore, since HMR can provide an increase in both the flexural load carrying capacity as well as the ductility, its use is recommended for beams treated with Boracol.

4.2. Creosoted Beams. As with the Boracol-treated beams, the use of SFRP significantly increased the ductility, toughness, and the load-carrying capacity (Figure 5 and Table 3 ). Surprisingly, the techniques seemed to perform even better on the Creosoted beams, as the magnitude of the increases were much greater than for the Boracol-treated beams. This was an encouraging result, as most structures in need of retrofit would likely be creosote treated or have some other sort of oil borne preservative treatment and would likely be weakened over time via natural processes. Of particular interest was the performance of Beam $G$ which only had been sprayed without any additional adhesive/bonding agent.

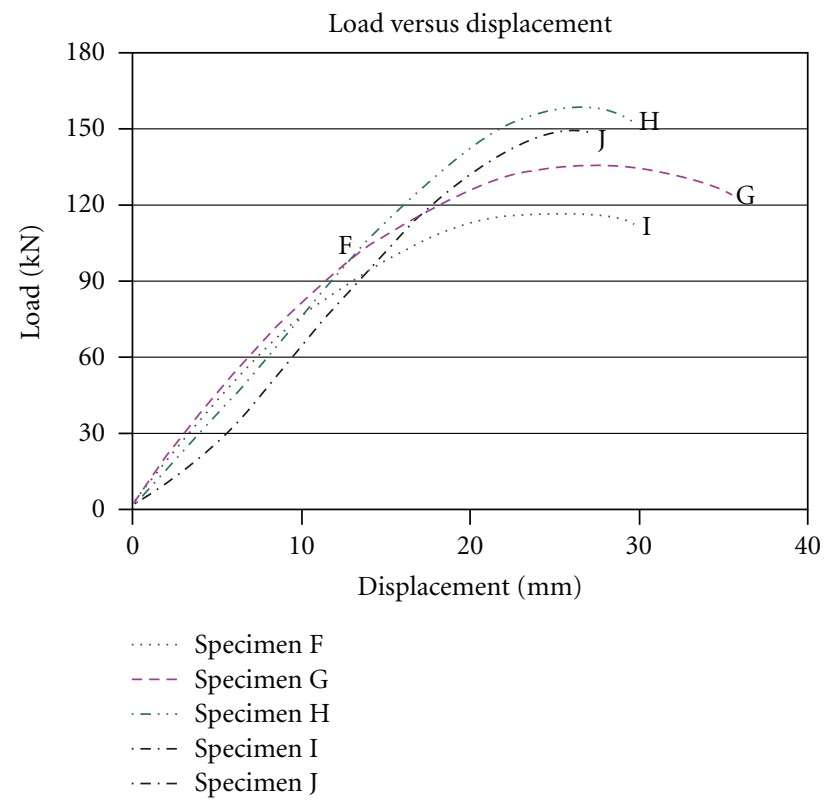

FIGURE 5: Comparison of results: creosoted.

Beam $G$ ended up displaying the highest energy increase and also showed a notable strength gain. This was in complete contrast to the Boracol treated specimens. Unlike the Boracol-treated specimens (Table 2), the HMR appeared to now have little effect in improving the SFRP-Timber bond. The AtPrime appeared to do a much better job of promoting adhesion between the two. The beam which was prepared with AtPrime only (Beam $\mathrm{H}$ ) showed the greatest increase in load capacity, and also provided an enormous increase in energy absorption.

The gain in ductility and energy absorption capacity afforded by the application of the SFRP is exceptional, and this characteristic could be utilized in applications such as seismic retrofit of structures or stiffening of structures against wind loading. The type of strength gain that was observed in the Creosoted specimens was at par with and in some cases exceeded those from previous similar retrofit experiments $[4,6,15]$. Given the promising results obtained, it is recommended that this characteristic be studied further and verified in an expanded testing program with additional variables considered, to confirm the observations from this project. 
TABLE 3: Summary of results: creosoted.

\begin{tabular}{lcccccc}
\hline & $\begin{array}{c}\text { Ultimate load } \\
(\mathrm{kN})\end{array}$ & $\begin{array}{c}\text { Ultimate displacement } \\
(\mathrm{mm})\end{array}$ & $\begin{array}{c}\text { Energy absorbed } \\
(\mathrm{kJ})\end{array}$ & $\begin{array}{c}\text { Load \% } \\
\text { increase over F }\end{array}$ & $\begin{array}{c}\text { Energy absorbed \% } \\
\text { increase over F }\end{array}$ & FRP rupture \\
\hline Beam F & 108.7 & 13.1 & 0.6 & - & - & - \\
Beam G & 139.3 & 35.6 & 3.6 & $28.2 \%$ & $460.1 \%$ & No \\
Beam H & 164.3 & 29.6 & 3.0 & $51.1 \%$ & $366.5 \%$ & No \\
Beam I & 112.4 & 29.9 & 2.5 & $3.4 \%$ & $287.4 \%$ & No \\
Beam J & 147.3 & 26.7 & 2.3 & $35.5 \%$ & $255.4 \%$ & Yes \\
\hline
\end{tabular}

\section{Discussion}

The bond between SFRP and timber appears to be the most important issue. To achieve maximum capacity of a retrofitted member, it is important that the FRP does not debond from the surface of the substrate to ensure its full utilization. SFRP and timber are two highly dissimilar materials — both chemically and mechanically-and debonding can easily occur at the interface between these two materials either due to environmental conditions (chemical contamination, volumetric expansion/contraction as a result of moisture movements) or due to buildup of shear stresses at the interface during loading. If the extent of delamination is substantial, the SFRP is unable to arrest the growth of fissures within the tensile zone of the beam, thereby precipitating a rapid beam failure. Even under less-thanideal bond conditions, the beam can still show significant strengthening and increases in ductility if the unloading of the tensile zone of the beam is delayed. Bond can be enhanced by ensuring the substrate surface is not contaminated, through the provision of a bonding agent, and by the use of mechanical anchorage between the FRP and substrate.

In addition to debonding as a result of dissimilar material moduli, test observations indicate that "free edge effects" and "face wrinkling" can both contribute to debonding. A laminate in tension will have a buildup of peel stresses at its free edge. Coupled with localized stresses which are generated in the vicinity of the loading plate, they may lead to premature peeling of the SFRP from the adherent surface. "Face wrinkling", on the other hand, occurs when the SFRP in the compression zone buckles and debonds from the adherent surface.

\section{Concluding Remarks}

Test results indicate that the application of Sprayed Fiber Reinforced Polymers (SFRPs) may be a promising technique for rehabilitation of timber beams. The technique may be particularly suited for creosoted beams where SFRP application demonstrated large gains in both the load-carrying capacity and ductility. For the Boracol-treated beams, while the technique is less effective, the use of HMR as the bonding agent can provide benefits. For contact-critical applications such as the one investigated here, a good bond between timber and SFRP is of critical importance. Every attempt should therefore be made to avoid or delay debonding.

\section{References}

[1] T. C. Triantafillou, "Composites: a new possibility for the shear strengthening of concrete, masonry and wood," Composites Science and Technology, vol. 58, no. 8, pp. 1285-1295, 1998.

[2] N. Banthia, N. Nandakumar, and A. J. Boyd, "Sprayed fiber reinforced polymers: from laboratory to a real bridge," ACI Concrete International: Design and Construction, vol. 24, no. 11, pp. 47-52, 2002.

[3] American Society of Civil Engineers, "Report Card for Americas Infrastructure 2005," Report Card for Americas Infrastructure, 2005, http://apps.asce.org/reportcard/2005/page .cfm? id=203.

[4] N. Plevris and T. Triantafillou, "FRP-reinforced wood as structural material," Journal of Materials in Civil Engineering, vol. 4, no. 3, pp. 300-317, 1992.

[5] C. Gentile, Flexural strengthening of timber bridge beams using FRP, M.S. thesis, University of Manitoba, Winnipeg, Canada, 2000.

[6] D. D. Dempsey and D. W. Scott, "Wood members strengthened with mechanically fastened FRP strips," Journal of Composites for Construction, vol. 10, no. 5, pp. 392-398, 2006.

[7] K. C. Johns and S. Lacroix, "Composite reinforcement of timber in bending," Canadian Journal of Civil Engineering, vol. 27, no. 5, pp. 899-906, 2000.

[8] J. Fiorelli and A. A. Dias, "Analysis of the strength and stiffness of timber beams reinforced with carbon fiber and glass fiber," Materials Research, vol. 6, no. 2, pp. 193-202, 2003.

[9] J. R. Gilfillan, S. G. Gilbert, and G. R. H. Patrick, "The use of FRP composites in enhancing the structural behavior of timber beams," Journal of Reinforced Plastics and Composites, vol. 22, no. 15, pp. 1373-1388, 2003.

[10] H. J. Dagher, T. E. Kimball, S. M. Shaler, and A. M. Beckry, "Effect of FRP reinforcement on low grade eastern hemlock glulams," in Proceedings of the National Conference on Wood in Transportation Structures, pp. 207-214, Madison, Wis, USA, 1996.

[11] H. Johnsson, T. Blanksvärd, and A. Carolin, "Glulam members strengthened by carbon fibre reinforcement," Materials and Structures/Materiaux et Constructions, vol. 40, no. 1, pp. 4756, 2007.

[12] C. A. Issa and Z. Kmeid, "Advanced wood engineering: glulam beams," Construction and Building Materials, vol. 19, no. 2, pp. 99-106, 2005.

[13] A. Dorey and J. Cheng, "The behavior of GFRP glue laminated wood beams," in Proceedings of the 2nd International Conference on Advanced Composite Materials in Bridges and Structures, pp. 787-794, Montreal, Canada, 1996.

[14] R. Hernandez, J. Davalos, S. Sonti, Y. Kim, and R. Moody, "Strength and stiffness of reinforced yellow- poplar gluedlaminated beams," Research Paper FPL-RP-553, USDA Forest Service Products Laboratory, Madison, Wis, USA, 1997. 
[15] C. Gentile, D. Svecova, W. Saltzberg, and A. Rizkalla, "Strengthening of timber bridges," in Proceedings of the Worldwise '99, Winnipeg, Canada, December 1999.

[16] A. A. Mufti, B. Bakht, D. Svecova, and T. Adams, "Evaluation of glue for bonding FRP laminates on timber beams treated with creosotes," in Proceedings of the 29th CSCE Annual Conference, Victoria, Canada, 2001.

[17] R. Martens, M. Siegel, and F. Linares, "Secondary bond strength of FRP to a variety of substrates," in Proceedings of the NACE Corrosion Symposium, Buffalo, NY, USA, 1996.

[18] C. B. Vick, "Hydroxymethylated resorcinol coupling agent for enhanced adhesion of epoxy and other thermosetting adhesives to wood," in Proceedings of the Wood Adhesives Symposium, pp. 47-55, Portland, Ore, USA, 1995.

[19] A. W. Christiansen and C. B. Vick, "Hydroxymethylated resorcinol coupling agent for wood surfaces to produce exterior durable bonds," Silanes and Other Coupling Agents, vol. 2, pp. 193-208, 2000.

[20] J. S. Lyons and M. R. Ahmed, "Factors affecting the bond between polymer composites and wood," Journal of Reinforced Plastics and Composites, vol. 24, no. 4, pp. 405-412, 2005.

[21] K. Richter and R. Steiger, "Thermal stability of woodwood and Wood-FRP bonding with polyurethane and epoxy adhesives," Advanced Engineering Materials, vol. 7, no. 5, pp. 419-426, 2005.

[22] N. Banthia and A. J. Boyd, "Sprayed fibre-reinforced polymers for repairs," Canadian Journal of Civil Engineering, vol. 27, no. 5, pp. 907-915, 2000.

[23] R. Solemani, Sprayed glass fiber reinforced polymers in shear strengthening and enhancement of impact resistance of reinforced concrete beams, Ph.D. thesis, University of British Columbia, Vancouver, Canada, 2006.

[24] J. D. Barrett and W. Lau, "Canadian lumber properties," in Canadian Wood Council, E. D. Jones, Ed., Canada Wood, Ottawa, Canada, 1994.

[25] S. Talukdar, Strengthing of timber beams using externally bonded sprayed fibre reinforced polymers, M.A.Sc thesis, University of British Columbia, Vancouver, Canada, 2008. 

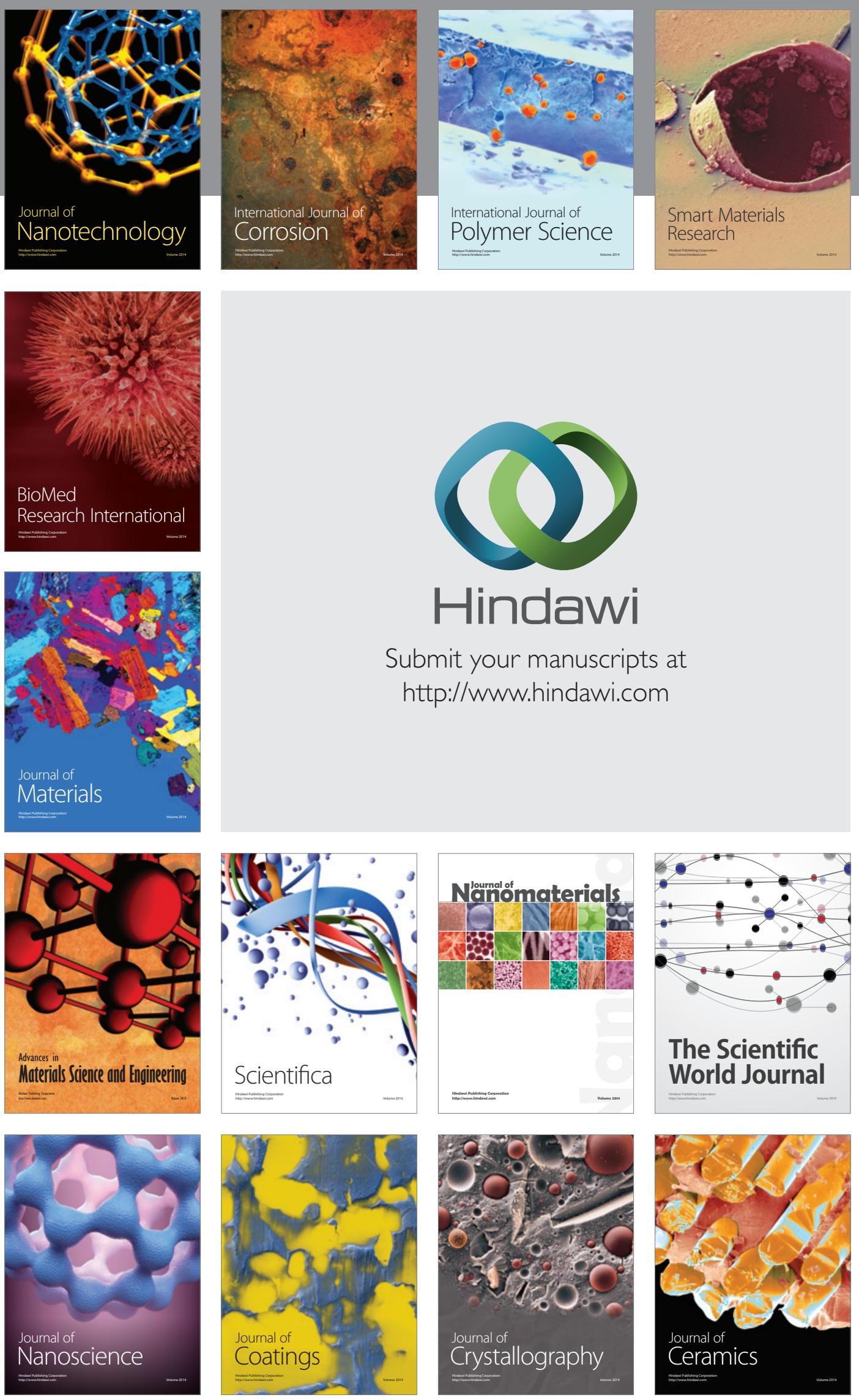

The Scientific World Journal

Submit your manuscripts at

http://www.hindawi.com

\section{World Journal}

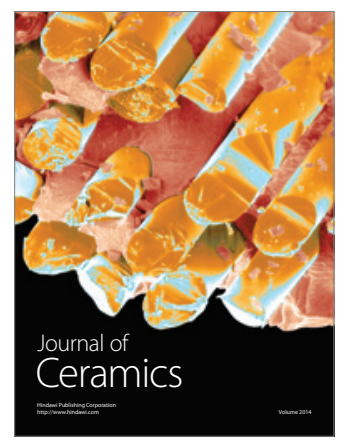

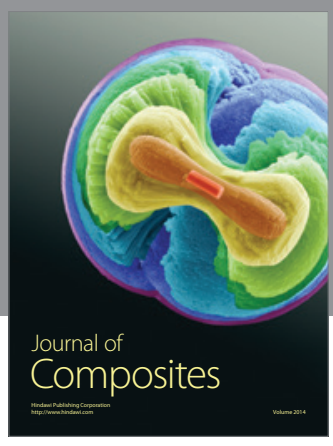
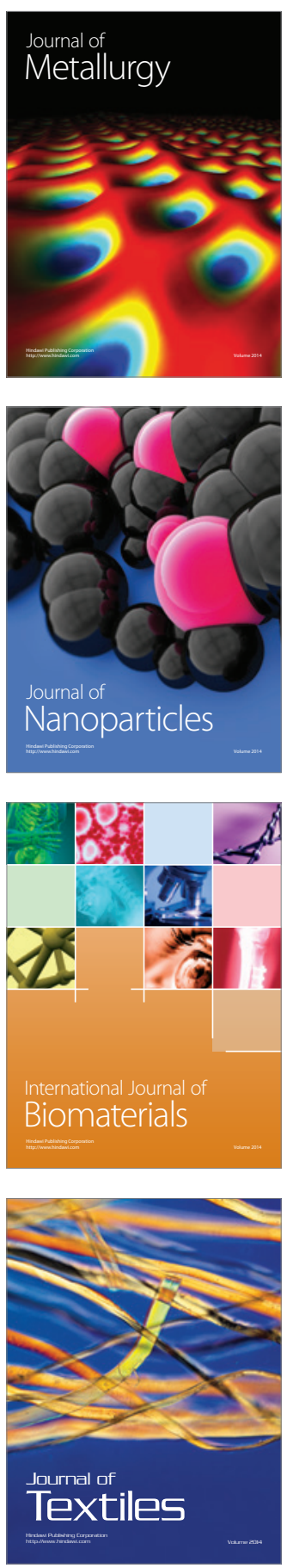